\title{
Tracking the Dynamics of an Ideal Quantum Measurement
}

\author{
Fabian Pokorny $\odot,{ }^{1, *}$ Chi Zhang $\odot,{ }^{1, \dagger}$ Gerard Higgins $\odot,{ }^{1, \$}$ Adán Cabello๑, ${ }^{2,3,8}$ \\ Matthias Kleinmann $\oplus^{4,5, \|}$ and Markus Hennrich $\oplus^{1, \pi}$ \\ ${ }^{1}$ Department of Physics, Stockholm University, 10691 Stockholm, Sweden \\ ${ }^{2}$ Departamento de Física Aplicada II, Universidad de Sevilla, E-41012 Sevilla, Spain \\ ${ }^{3}$ Instituto Carlos I de Física Teórica y Computacional, Universidad de Sevilla, E-41012 Sevilla, Spain \\ ${ }^{4}$ Department of Theoretical Physics, University of the Basque Country UPV/EHU, E-48080 Bilbao, Spain \\ ${ }^{5}$ Naturwissenschaftlich-Technische Fakultät, Universität Siegen, 57068 Siegen, Germany
}

(Received 28 March 2019; revised manuscript received 4 September 2019; accepted 24 January 2020; published 25 February 2020; corrected 4 March 2020)

\begin{abstract}
The existence of ideal quantum measurements is one of the fundamental predictions of quantum mechanics. In theory, an ideal measurement projects a quantum state onto the eigenbasis of the measurement observable, while preserving coherences between eigenstates that have the same eigenvalue. The question arises whether there are processes in nature that correspond to such ideal quantum measurements and how such processes are dynamically implemented in nature. Here we address this question and present experimental results monitoring the dynamics of a naturally occurring measurement process: the coupling of a trapped ion qutrit to the photon environment. By taking tomographic snapshots during the detection process, we show that the process develops in agreement with the model of an ideal quantum measurement with an average fidelity of $94 \%$.
\end{abstract}

DOI: 10.1103/PhysRevLett.124.080401

Introduction.-What is an ideal measurement in quantum mechanics? What are its inner workings? How does the quantum state change because of it? These have been central questions in the development of quantum mechanics [1]. Notably, today's accepted answer to the latter question is conceptually different from the one given in the first formalization of quantum mechanics by von Neumann [2]. Then, it was thought that an ideal measurement on a quantum system would inevitably destroy all quantum superpositions. Later, Lüders pointed out [3] that certain superpositions should survive, so that a sequence of ideal measurements would preserve quantum coherence. Lüders's version is the one accepted today.

Even though there is agreement on the theoretical description of an ideal measurement, it is a fundamental question whether, and, if so, how ideal measurements occur as natural processes. This is an especially sensitive question, as measurements do not fall into the domain of unitary time evolution. In this Letter, we demonstrate a natural process that is considered to be an ideal measurement and monitor its dynamics by taking a sequence of snapshots while the process is occurring. These snapshots are tomographically complete and allow us to compare the

Published by the American Physical Society under the terms of the Creative Commons Attribution 4.0 International license. Further distribution of this work must maintain attribution to the author(s) and the published article's title, journal citation, and DOI. Funded by Bibsam. experimental results with the theoretical prediction of an ideal measurement. As the natural process, we choose a fluorescence measurement of a trapped atomic ion in contrast to a fine-tuned process in an artificially created system [4]. The time evolution of the process then provides insight into how the measurement process occurs naturally.

Ideal measurements model an ideal implementation of a quantum observable. In the case of a discrete spectrum, a measurement of the observable $A$ yields values $a_{k}$ according to the spectral decomposition $A=\sum_{k} a_{k} \Pi_{k}$, where $\Pi_{k}$ are mutually orthogonal projections summing to identity, and $a_{k}$ are distinct real numbers. Any measurement requires an interaction of the measurement apparatus with the system and this interaction affects the state of the system. This is true, independent of whether or not the measurement result is recorded by an observer. However, the effect on the state depends on the experimental realization. In practice, a measurement is often a rather violent process that "destroys" the quantum system, for example, the detection of a photon.

A Lüders process is the ideal implementation of a measurement in which the state of the system is transformed according to [5]

$$
\Xi_{A}: \rho \mapsto \sum_{k} \Pi_{k} \rho \Pi_{k} .
$$

(For simplicity, we assume that the observer ignores the measurement outcome.) From a theoretical perspective, this process is truly special. On the one hand, it is the only process implementing a measurement of $A$ that does not disturb any subsequent refined measurement $B[6,7]$; that 
is, a measurement $B$ where each outcome $\ell$ is at most as likely as a certain outcome $k_{\ell}$ of $A$, for all states $\rho$. This implies that, whenever two observables $A$ and $B$ are compatible, $A B=B A$, then the respective Lüders processes do not disturb each other, $\Xi_{A} \Xi_{B}=\Xi_{B} \Xi_{A}[3,5]$. On the other hand, $\Xi_{A}$ is universal: any process $\Lambda_{A}$ describing a measurement of $A$ can be written as $\Lambda_{A}: \rho \mapsto$ $\sum_{k} \Phi_{k}\left(\Pi_{k} \rho \Pi_{k}\right)$, where $\Phi_{k}$ are some probability-preserving processes [5].

While these properties constitute strong theoretical arguments for the special role of the Lüders process, this does not imply that Lüders processes occur in nature, and it does not tell us how nature implements them. An important aspect of the Lüders process is that it leaves any quantum superposition of degenerate eigenstates unaffected. In this sense, the existence of Lüders processes is a nontrivial prediction of quantum mechanics that is usually taken for granted rather than analyzed thoroughly.

The structure of a dynamical realization of a Lüders process is best understood by considering the canonical model for measurements [5,8]: A system in state $\rho$ is brought into contact with a pointer system, which is in state $|\Phi\rangle$. When in contact, the two systems interact via the Hamiltonian $H_{A}$ for a time $\tau$ and, after separating the two systems, the pointer system is measured in some fixed basis $\left|\omega_{j}\right\rangle$. This yields the process

$$
M_{A}: \rho \mapsto \sum_{j}\left\langle\omega_{j}\left|e^{-i H_{A} \tau / \hbar}(\rho \otimes|\Phi\rangle\langle\Phi|) e^{i H_{A} \tau / \hbar}\right| \omega_{j}\right\rangle .
$$

However, the class of processes covered by Eq. (2) is so general that it can be shown [5] that any quantum process, including the Lüders processes $\Xi_{A}$, can be modeled by Eq. (2). Indeed, if we are able to engineer interactions and pointer systems at will, as in, for example, quantum processors [9,10], the fact that a process cannot be approximated by Eq. (2) would be in contradiction of our present understanding of those systems. Usually, experimental measurement procedures are not Lüders processes, because this would require a careful fine-tuning of the Hamiltonian $H_{A}$ or else the degeneracy of the observable would be lifted by experimental imperfections, which then leads to a process as the one envisioned by von Neumann, in which all coherences are destroyed.

For example, in a recent experiment [4], a Lüders-type process was implemented on an artificial three-level quantum system with an overall fidelity of $94 \%$. The interaction to the pointer state (stored in a microwave field transmitted through a cavity) was tuned in such a way that the coherence in a two-dimensional subspace was preserved.

In this Letter, we use a fluorescence measurement on an ion to implement a Lüders process. Since the Hamiltonian is given by the natural interaction between the ion and the photon field, we are then particularly interested in the dynamics that leads to the implementation of the Lüders process.

Fluorescence measurements.-The best candidate for a natural Lüders process is an interaction-free measurement, that is, a measurement of an observable of the form $A=|\phi\rangle\langle\phi|$, where the event "detection" corresponds to the eigenvalue 1 and the event "no detection" corresponds to the eigenvalue 0 . There, it can be expected that the nodetection event does not require any interaction with the eigenspace with eigenvalue 0 , and hence superpositions in this subspace are preserved. Our choice for an experiment within which to identify a Lüders process is the coupling of a single trapped ion to the photon environment. To explain why this is a good candidate, it is useful to present a simplified description of the specific physical process in our experiment. For a more accurate theoretical model of this process, see Ref. [11] and the Supplemental Material [12].

We prepare an ion in a superposition of three electronic states: $|0\rangle,|1\rangle$, and $|2\rangle$. By driving the transition $|0\rangle$ to a short-lived excited level $|e\rangle$, we facilitate the emission of photons into the environment, via the natural decay $|e\rangle|n=0\rangle \rightarrow|0\rangle|n=1\rangle$. Here, $|n=0\rangle$ is the initial state of the photon environment and $|n=1\rangle$ is the state of the photon environment after a photon has been emitted by the decay. We write the initial state of the system and the photon environment as

$$
|\Psi\rangle=\left(\alpha_{0}|0\rangle+\alpha_{1}|1\rangle+\alpha_{2}|2\rangle\right)|n=0\rangle .
$$

Since only the level $|0\rangle$ participates in the driving $|0\rangle \leftrightarrow|e\rangle$ and the subsequent decay, the state after the driving is

$$
\begin{aligned}
\left|\Psi_{m}\right\rangle= & \alpha_{0}|0\rangle\left(g_{0}|n=0\rangle+g_{1}|n=1\rangle\right) \\
& +\left(\alpha_{1}|1\rangle+\alpha_{2}|2\rangle\right)|n=0\rangle,
\end{aligned}
$$

where $g_{1}$ is related to the probability of photon scattering by $P_{\text {scatt }}=\left|g_{1}\right|^{2}$, and $\left|g_{0}\right|^{2}+\left|g_{1}\right|^{2}=1$. Ignoring the photon environment, we obtain the reduced state

$$
\rho_{m}=\left(\begin{array}{ccc}
\left|\alpha_{0}\right|^{2} & \alpha_{0} \alpha_{1}^{*} g_{0} & \alpha_{0} \alpha_{2}^{*} g_{0} \\
\alpha_{0}^{*} \alpha_{1} g_{0}^{*} & \left|\alpha_{1}\right|^{2} & \alpha_{1} \alpha_{2}^{*} \\
\alpha_{0}^{*} \alpha_{2} g_{0}^{*} & \alpha_{1}^{*} \alpha_{2} & \left|\alpha_{2}\right|^{2}
\end{array}\right) .
$$

The coherence between levels $|1\rangle$ and $|2\rangle$ is preserved, while the coherences between $|0\rangle$ and $|1\rangle$ and between $|0\rangle$ and $|2\rangle$ decay with $\left|g_{0}\right|$.

Intuitively, if at least one photon is scattered into the environment, then $\left|g_{0}\right|^{2}=0$ and the coupling to the photon environment corresponds to the measurement of the qutrit projection $(|0\rangle\langle 0|, \mathbb{1}-| 0\rangle\langle 0|)$. In general, $g_{0}$ depends on the strength of the interaction and, for a weak or short interaction, $\left|g_{0}\right|^{2}$ is between zero and one. These intermediate values are then given by the evolution leading to the Lüders process. Because the probability for scattering is 
$P_{\text {scatt }}=1-\left|g_{0}\right|^{2}$, the implemented measurement is the generalized measurement $\left(E_{1}, E_{0}\right)$ with $E_{1}=P_{\text {scatt }}|0\rangle\langle 0|$ and $E_{0}=\mathbb{1}-E_{1}$. The numerical value of $g_{0}$ depends on the experimental configuration, and its computation requires a more rigorous model of the measurement process. A formula for $g_{0}$ is given in Eq. (7) below and the interaction strength is varied in the experiment such that $P_{\text {scatt }}$ takes values between 0.33 and 1.0.

Since our computation of the state $\rho_{m}$ is generic for any initial state, we can read off the measurement process $\Lambda_{m}$ as

$$
\Lambda_{m}: \rho \mapsto \sqrt{E_{1}} \rho \sqrt{E_{1}}+G \sqrt{E_{0}} \rho \sqrt{E_{0}} G^{\dagger},
$$

with $G=e^{i \varphi}|0\rangle\langle 0|+| 1\rangle\langle 1|+| 2\rangle\langle 2|$ and $\varphi=\arg \left(g_{0}\right)$. For $P_{\text {scatt }} \rightarrow 1$, this process implements the Lüders process $\Xi_{A}$ for the observable $A=|0\rangle\langle 0|$ and hence constitutes an ideal measurement. For $P_{\text {scatt }}<1$, the process is a transitional form between $\Xi_{A}$ and the trivial process $\rho \mapsto \rho$. Then, not only is the coherence between $|1\rangle$ and $|2\rangle$ preserved, but also partially the coherences between $|0\rangle$ and the other two qutrit levels. The occurrence of the terms $\sqrt{E_{k}} \rho \sqrt{E_{k}}$ in $\Lambda_{m}$ is in accordance with the canonical form of such transitional processes [5], while the phase $e^{i \varphi}$ introduced by the unitary $G$ is specific to this measurement process.

Experimental setup.-Our setup consists of a single ${ }^{88} \mathrm{Sr}^{+}$ ion confined in a linear Paul trap, similar to Ref. [13]. Figure 1(a) shows the level scheme of the ion with the qutrit states encoded in electronic states of the ion. The measurement process is implemented using a pulse of $422 \mathrm{~nm}$ laser light, which facilitates coupling of the electronic state $|0\rangle$ and the photon environment, while the qutrit levels $|1\rangle$ and $|2\rangle$ are left unaffected. We use the same laser light during the fluorescence detection step.

The variable $g_{0}$ is obtained from a quantum optical model of the measurement (see the Supplemental Material [12]). This yields

$$
g_{0} \approx \exp \left(-\frac{\Omega^{2}}{2 \Gamma+4 i \Delta} t\right) \cdot \exp \left(i \phi_{r}\right),
$$

where $\Omega$ is the Rabi frequency driving the $|0\rangle \leftrightarrow|e\rangle$ transition, $\Gamma=2 \pi \times 21.65 \mathrm{MHz}$ [14] is the decay rate of state $|e\rangle, \Delta=2 \pi \times(5 \pm 2) \mathrm{MHz}$ is the detuning from resonance, $t=1 \mu \mathrm{s}$ is the length of the laser pulse, and the phase $\phi_{r}$ results from the ac-Stark shifts induced by repump laser fields at 422 and $1092 \mathrm{~nm}$, which are present during the measurement process. The intensities and detunings of the repump fields are tuned such that $\operatorname{Im}\left(g_{0}\right) \approx 0$ (see Supplemental Material [12]). Values of $\Omega$ and $\Delta$ are determined from the $5 S_{1 / 2} \leftrightarrow 5 P_{1 / 2}$ spectral line shape.

We track the dynamics of the measurement process by carrying out process tomography as the power of the $422 \mathrm{~nm}$ laser used for coupling $|0\rangle \leftrightarrow|e\rangle$ is varied. For the process tomography [Fig. 1(b)], the ion is prepared in $|0\rangle$, then rotated (unitary $U_{i}$ ) to one of the nine initial

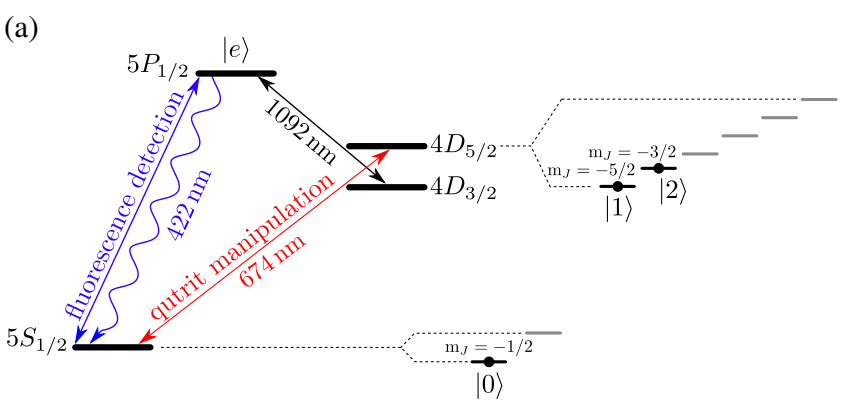

(b)



FIG. 1. Experimental realization of the process tomography of a Lüders measurement. (a) Level scheme of ${ }^{88} \mathrm{Sr}^{+}$. A magnetic field of $0.36 \mathrm{mT}$ applied along the trap axis splits the $m_{J}=-5 / 2$ and $m_{J}=-3 / 2$ Zeeman sublevels of the metastable states $4 D_{5 / 2}$ by $2 \pi \times 6.0 \mathrm{MHz}$. Together with the $5 S_{1 / 2} m_{J}=-1 / 2$ ground state, these states form the qutrit basis states $|0\rangle,|1\rangle$, and $|2\rangle$. The qutrit transitions are driven by a narrow-linewidth laser at $674 \mathrm{~nm}$ (linewidth $\langle 2 \pi \times 600 \mathrm{~Hz}$ ) tuned to either the $\mid 0\rangle \leftrightarrow|1\rangle$ or $|0\rangle \leftrightarrow|2\rangle$ transition. A $422 \mathrm{~nm}$ laser driving the $|0\rangle \leftrightarrow|e\rangle \equiv$ $5 P_{1 / 2}$ transition is used to induce the Lüders measurement process and the fluorescence detection during state tomography. To prevent loss of population from the qutrit subspace during the measurement process, $1092 \mathrm{~nm}$ laser light and circularly polarized $422 \mathrm{~nm}$ laser light are used to repump population from $4 D_{3 / 2}$ and $5 S_{1 / 2} m_{J}=+1 / 2$ to $|0\rangle$. (b) Experimental sequence for the process tomography. First, the ion is prepared in one of nine initial states (see Supplemental Material [12]) followed by a measurement that implements snapshots of an ideal measurement process, and finally, state tomography is performed using a qutrit rotation and fluorescence detection.

states $\left|\psi_{i}\right\rangle$ using pulses of $674 \mathrm{~nm}$ laser light (see the Supplemental Material [12]). Then the measurement process is carried out; a $422 \mathrm{~nm}$ laser pulse is applied for $1 \mu \mathrm{s}$. Finally, the state tomography is carried out by applying rotation $U_{j}^{\dagger}$ followed by fluorescence detection for $500 \mu \mathrm{s}$. The measurement process uses a shorter pulse length than the fluorescence detection step, since the photons scattered during the measurement process do not need to be detected. During state tomography the rotation $U_{j}^{\dagger}$ followed by a measurement of $(|0\rangle\langle 0|, \mathbb{1}-| 0\rangle\langle 0|)$ acts as measurement operator $\left(\left|\psi_{j}\right\rangle\left\langle\psi_{j}|, \mathbb{1}-| \psi_{j}\right\rangle\left\langle\psi_{j}\right|\right)$. The nine initial states are each measured by the nine different operators; this is sufficient to characterize the experimental process.

Results.-For analyzing the experiment, a process $\Lambda$ is conveniently represented by its Choi matrix [5],

$$
\chi=\sum_{i, j=0}^{2} \Lambda\left[| i \rangle \langle j | _ { \mathrm { sys } } ] \otimes | i \rangle \left\langle\left.j\right|_{\mathrm{aux}} .\right.\right.
$$




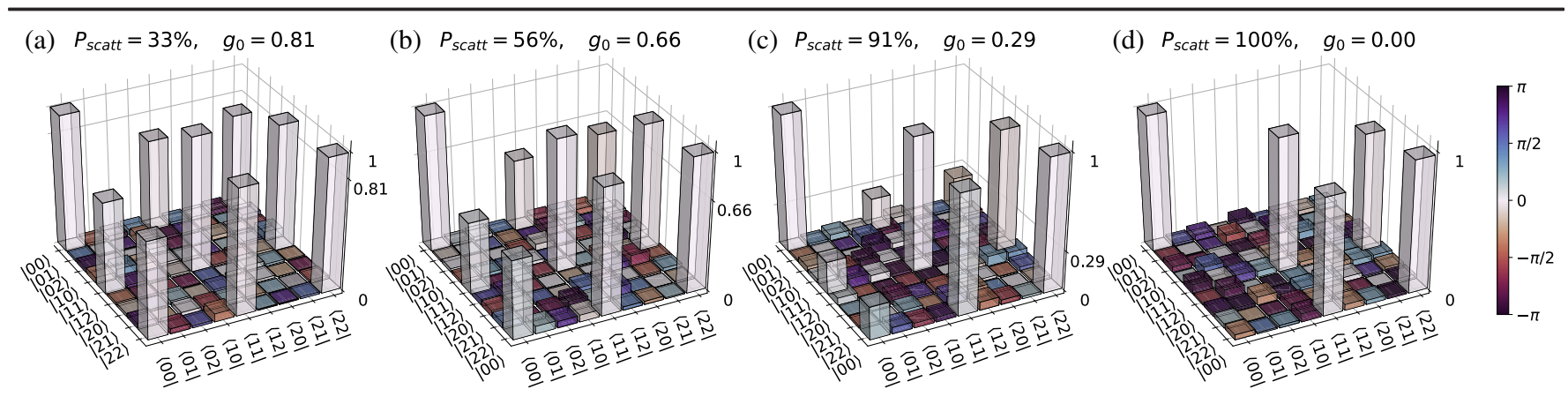

FIG. 2. Choi matrices $\chi_{\exp }$ reconstructed from the experimental data. The colors indicate the phase, $g_{0}$ is calculated according to our model, see Eq. (7). (a)-(d) The coupling strength of $|0\rangle$ to the photon environment is increased and coherences involving state $|0\rangle$ are destroyed. (d) Similar to the ideal Lüders process, and from (d) $\rightarrow$ (a) the process becomes more similar to the trivial process $\rho \mapsto \rho$.

This matrix is positive semidefinite for any physical process and yields a complete characterization of $\Lambda$, since $\Lambda[\rho]=\operatorname{tr}_{\text {aux }}\left[\chi\left(\mathbb{1} \otimes \rho^{T}\right)\right]$ with $\rho^{T}=\sum_{i, j}|i\rangle\langle j|\langle j|\rho| i\rangle$. If $\Lambda$ is a probability-preserving process, that is, $\operatorname{tr}(\Lambda[\rho])=$ $\operatorname{tr}(\rho)=1$, then the Choi matrix obeys $\operatorname{tr}_{\text {sys }}(\chi)=\mathbb{1}_{\text {aux }}$. For the measurement process $\Lambda_{m}$ in this Letter, the Choi matrix reads

$$
\chi_{m}=|00\rangle\langle 00|+| \xi\rangle\left\langle\xi\left|+g_{0}\right| 00\right\rangle\left\langle\xi\left|+g_{0}^{*}\right| \xi\right\rangle\langle 00|,
$$

where $|\xi\rangle=|11\rangle+|22\rangle$.

The system is prepared in $\left|\psi_{i}\right\rangle$, the process $\Lambda_{m}$ is carried out, and subsequently, the system is measured using the observable $\left|\psi_{j}\right\rangle\left\langle\psi_{j}\right|$. This is repeated $N=1000$ times for each $i, j$, with measurement outcome 1 occurring $n_{i, j}$ times, and thus resulting in the experimental outcome frequency $f_{i, j}=n_{i, j} / N$. We use $f_{i, j}$ to reconstruct the experimental Choi matrix $\chi_{\exp }$ with $\chi_{\exp }=\arg \min _{\chi} \sum_{i, j}\left(p_{i, j}-f_{i, j}\right)^{2}$, where the probabilities $p_{i, j}$ are given by

$$
\begin{aligned}
p_{i, j} & =\left\langle\psi_{j}\left|\Lambda\left[\left|\psi_{i}\right\rangle\left\langle\psi_{i}\right|\right]\right| \psi_{j}\right\rangle \\
& =\operatorname{tr}\left[\chi\left|\psi_{j}\right\rangle\left\langle\psi_{j}|\otimes| \psi_{i}\right\rangle\left\langle\left.\psi_{i}\right|^{T}\right] .\right.
\end{aligned}
$$

In Fig. 2, we show a graphical representation of the different snapshots, namely, at about 33\%, 56\%, 91\%, and $100 \%$ of the maximal measurement interaction strength. For the weakest interaction, the process is very similar to the trivial channel (with only $\langle i i|\chi| j j\rangle=1$ ). With increasing strength of the measurement interaction, the terms responsible for preserving the coherence between $|0\rangle$ and $|1\rangle$, and $|0\rangle$ and $|2\rangle$ (i.e., $\langle 11|\chi| 00\rangle$ and $\langle 22|\chi| 00\rangle$ ), decay more and more. This shows that, with increasing interaction strength, the coherence between $|0\rangle$ and $|1\rangle$ is more and more destroyed, while any superposition of $|1\rangle$ and $|2\rangle$ remains mostly unaltered. For further illustration, we present the evolution of the quantum states $(1 / \sqrt{2})(|1\rangle+i|2\rangle)$ and $(1 / \sqrt{2})(|0\rangle+i|2\rangle)$ in Fig. 3 of the Supplemental Material [12]. There, the loss of the coherence involving $|0\rangle$ and the preservation of the coherence between $|1\rangle$ and $|2\rangle$ can be observed directly.

In addition, we calculate the Uhlmann fidelity (rescaled by $\frac{1}{9}$ ) of the experimental Choi matrix and the model to be, on average, 94\%. For details, see the Supplemental Material [12].

$\chi_{\exp }$ does not perfectly obey the probability-preserving constraint $\operatorname{tr}_{\text {sys }} \chi=\mathbb{1}_{\text {aux }}$, due to statistical fluctuations and imperfections in the experimental setup. A likelihood ratio test shows that the deviation from $\operatorname{tr}_{\mathrm{sys}} \chi=\mathbb{1}_{\mathrm{aux}}$ is statistically significant by between 4 and 8 standard deviations. This can be attributed to imperfect state preparation. While the effect is statistically significant, it is clear from the high number of samples that the systematic deviation is overall very small and can be neglected. A comparison of the $\chi_{\exp }$ reconstructed from experimental data with our model predictions, including the respective uncertainties, can be found in the Supplemental Material [12].

Conclusion.-Fluorescence detection is the standard way to measure a qubit in an ion trap or in similar setups that enable quantum information processing. This process is a prime example of an ideal measurement process: The system that is subject to the measurement is brought into contact with a macroscopic pointer state by facilitating a strong interaction between state $|0\rangle$ of the system and the photon environment. A measurement of the photon environment then reveals the state of the system. The predictions of quantum mechanics for ideal measurements become apparent when a qutrit is employed: Any coherence between states $|1\rangle$ and $|2\rangle$, which are not coupled to the photon environment, should persist. We verified this property by performing process tomography of the coupling of electronic states of a single trapped ion to the photon environment.

The theoretically ideal measurement process only occurs in the case of a strong interaction. If the interaction is weakened, then an intermediate process between the ideal measurement process and the identity process occurs. In the mathematical theory of measurements [5], such ideal measurements have a canonical "square root" form; see 
Eq. (6) with $G=\mathbb{1}$. The quantum optical model for a weak measurement predicts a similar form. In process tomographies for those intermediate processes, we obtain an overall process fidelity with the predicted model of approximately $94 \%$.

We thus quantitatively demonstrated how a measurement can be implemented in a natural process while preserving coherence. By taking snapshots of the measurement process itself, we also demonstrated that this process is not instantaneous. A similar conclusion was reached in [15], in which it was found that quantum jumps evolve coherently and are not instantaneous. A future direction of research is to test the predictions of ideal quantum measurements beyond what we have tested here: In the current experiment, the coherence-preserving measurement works relatively effortlessly, since the measurement process only affects a single state of our qutrit. This corresponds to an interaction-free measurement, where it is only measured whether or not the system is in state $|0\rangle$. It could also be possible to find an ideal measurement process in nature, where all eigenvalues of the measured observable are twofold degenerate, and therefore, the coherence between the degenerate eigenstates needs to be preserved. Whether such processes exist as natural processes and can be implemented with a fidelity comparable to our experiment is an open question.

We thank Mohamed Bourennane and Markus Müller for discussions. This work was supported by the Spanish Ministry of Economy, Industry and Competitiveness MINECO and the European Regional Development Fund FEDER: European Regional Development Fund through Grants No. FIS2017-89609-P and No. FIS2015-67161-P, by the FQXi Large Grant "The Observer Observed: A Bayesian Route to the Reconstruction of Quantum Theory," by the EU (ERC Consolidator Grant No. 683107/TempoQ and ERC Starting Grant No. 258647/GEDENTQOPT), by the Basque Government (Grant No. IT986-16), by the Swedish Research Council (Trapped Rydberg Ion Quantum Simulator), by QuantERA ERA-NET Cofund in Quantum Technologies implemented within the European Union's Horizon 2020 Programme (ERyQSenS), and by the project "Photonic Quantum Information" (Knut and Alice Wallenberg Foundation, Sweden).

*fabian.pokorny@fysik.su.se

†chi.zhang@fysik.su.se

${ }^{\ddagger}$ gerard.higgins@fysik.su.se

§adan@us.es

"matthias.kleinmann@uni-siegen.de

"markus.hennrich@fysik.su.se

[1] Quantum Theory and Measurement, edited by J. A. Wheeler and W. H. Zurek (Princeton University Press, Princeton, NJ, 1983).
[2] J. von Neumann, Mathematische Grundlagen der Quantenmechanik (Springer-Verlag, Berlin, 1932); [Enlish translation Mathematical Foundations of Quantum Mechanics (Princeton University Press, Princeton, NJ, 1955)], in particular, Chap. 5, Sec. I.

[3] G. Lüders, Über die Zustandsänderung durch den Meßprozeß, Ann. Phys. (Berlin) 443, 322 (1950); [English translation Concerning the state-change due to the measurement process, Ann. Phys. (Berlin) 15, 663 (2006)].

[4] M. Jerger, P. Macha, A. R. Hamann, Y. Reshitnyk, K. Juliusson, and A. Fedorov, Realization of a Binary-Outcome Projection Measurement of a Three-Level Superconducting Quantum System, Phys. Rev. Applied 6, 014014 (2016).

[5] T. Heinosaari and M. Ziman, The Mathematical Language of Quantum Theory: From Uncertainty to Entanglement (Cambridge University Press, Cambridge, England, 2012).

[6] G. Chiribella and X. Yuan, Measurement sharpness cuts nonlocality and contextuality in every physical theory, arXiv:1404.3348.

[7] M. Kleinmann, Sequences of projective measurements in generalized probabilistic models, J. Phys. A 47, 455304 (2014).

[8] A. Peres, Quantum Theory: Concepts and Methods Kluwer (Kluwer, Dordrecht, 1995).

[9] A. Barenco, C. H. Bennett, R. Cleve, D. P. DiVincenzo, N. Margolus, P. Shor, T. Sleator, J. A. Smolin, and H. Weinfurter, Elementary gates for quantum computation, Phys. Rev. A 52, 3457 (1995).

[10] D. Deutsch, Quantum theory, the Church-Turing principle and the universal quantum computer, Proc. R. Soc. A 400, 97 (1985).

[11] H. Carmichael, An Open Systems Approach to Quantum Optics (Springer-Verlag, Berlin, 1993).

[12] See Supplemental Material at http://link.aps.org/ supplemental/10.1103/PhysRevLett.124.080401 for further details on the model of the measurement process, the experimental settings, and the initial state preparation, as well as a comparison of the $\chi_{\exp }$ reconstructed from experimental data with our model predictions and the postprocess density matrices for the initial states $(1 / \sqrt{2})(|1\rangle+i|2\rangle)$ and $(1 / \sqrt{2})(|0\rangle+i|2\rangle)$.

[13] G. Higgins, W. Li, F. Pokorny, C. Zhang, F. Kress, C. Maier, J. Haag, Q. Bodart, I. Lesanovsky, and M. Hennrich, Single Strontium Rydberg Ion Confined in a Paul Trap, Phys. Rev. X 7, 021038 (2017).

[14] A. Gallagher, Oscillator strengths of Ca II, Sr II, and Ba II, Phys. Rev. 157, 24 (1967).

[15] Z. K. Minev, S. O. Mundhada, S. Shankar, P. Reinhold, R. Gutiérrez-Jáuregui, R. J. Schoelkopf, M. Mirrahimi, H. J. Carmichael, and M. H. Devoret, To catch and reverse a quantum jump mid-flight, Nature (London) 570, 200 (2019).

Correction: The license statement contained an omission and has been fixed. 